\title{
Accurate Detection of Paediatric Ventricular Tachycardia in AED
}

\author{
U. Irusta, E. Aramendi, J. Ruiz and S. Ruiz de Gauna \\ University of the Basque Country \\ Spain
}

\section{Introduction}

Sudden cardiac death (SCD) is the single most important cause of death in the adult population of the industrialized world (Jacobs et al., 2004). SCD accounts for 100-200 deaths per 100000 adults over 35 years of age annually (Myerburg, 2001). The most frequent cause of SCD is fatal ventricular arrhythmias: ventricular fibrillation (VF) and ventricular tachycardia (VT) (de Luna et al., 1989). In fact, degenerating VT to VF appears to be the mechanism for a large majority of cardiac arrests (Luu et al., 1989).

Most sudden cardiac arrests occur out of hospital, and the annual incidence of out-of-hospital cardiac arrest (OHCA) treated by emergency medical services in the USA is 55 per 100000 of the population (Myerburg, 2001). Survival rates in untreated cardiac arrest decrease by $7-10 \%$ per minute (Larsen et al., 1993; Valenzuela et al., 1997), as the heart function deteriorates rapidly. Consequently, early intervention is critical for the survival of OHCA victims. The sequence of actions to treat OHCA includes rapid access to emergency services, early cardiopulmonary resuscitation, early defibrillation and advanced cardiac life support as soon as possible. These four links constitute what the American Heart Association (AHA) calls the chain of survival. Compressions and ventilations during cardiopulmonary resuscitation maintain a minimum blood flow until defibrillation is available. The only effective way to terminate lethal ventricular arrhythmias and to restore a normal cardiac rhythm is defibrillation, through the delivery of an electric shock to the heart.

Automated external defibrillators (AED) are key elements in the chain of survival. An AED is a portable, user-friendly device that analyzes the rhythm acquired through two electrode pads and delivers an electric shock if pulseless VT or VF is detected. The shock advice algorithm (SAA) of an AED analyzes the electrocardiogram (ECG) to discriminate between shockable and nonshockable rhythms. Given a database of classified ECG records, the performance of the SAA is evaluated in terms of the proportion of correctly identified shockable—sensitivity—and nonshockable—specificity—rhythms, which must exceed the minimum values set by the AHA (Kerber et al., 1997).

SCD is 10 times less frequent in children than in adults. However, the social and emotional impact of the death of a child is enormous. In the USA alone, an estimated 16000 children die each year from sudden cardiac arrest (Sirbaugh et al., 1999). The most common cause of cardiac arrest in children is respiratory arrest, although the incidence of cardiac arrest caused by ventricular arrhythmias increases with age (Appleton et al., 1995; Atkins et al., 
1998). Initially two independent studies provided evidence for the use of AED in paediatric patients (Atkinson et al., 2003; Cecchin et al., 2001). Based on that evidence, in 2003, the International Liaison Committee on Resuscitation (ILCOR) recommended the use of AED in children aged 1-8 years (Samson et al., 2003). Since 2005, the resuscitation guidelines ${ }^{1}$ have incorporated this recommendation, which indicates the need to adapt AED for paediatric use. This adaptation involves adjusting the defibrillation pads and energy dose, and, furthermore, demonstrating that SAA accurately detect paediatric arrhythmias.

SAA of commercial AED were originally developed for adult patients. Adapting these algorithms for paediatric use requires the compilation of shockable and nonshockable rhythms from paediatric patients in order to assess their SAA performance. The first studies on the use of AED in children showed that two adult SAAs from commercial AED accurately identified many paediatric rhythms (Atkinson et al., 2003; Cecchin et al., 2001). The specificity for nonshockable rhythms and the sensitivity for VF were above the values recommended by the AHA. However, those studies failed to meet AHA criteria for shockable paediatric VT. In 2008, a third study showed that a SAA designed for adult patients did not meet AHA criteria for nonshockable paediatric supraventricular tachycardia (SVT) (Atkins et al., 2008). This study suggested that the specificity of SAA originally designed for adult patients could fail to meet AHA performance goals for paediatric SVT.

The SAA first extracts a set of discrimination parameters or features from the surface ECG recorded by the AED, and then combines those features to classify the rhythm as shockable or nonshockable. There are important differences in heart rate, amplitude and ECG wave morphology between paediatric and adult rhythms (Cecchin et al., 2001; Rustwick et al., 2007). The faster heart rates and shorter QRS durations of paediatric rhythms produce differences in the values of the discrimination parameters which may affect the performance of SAA designed for adult use. Some of these differences have been previously assessed, and the discrimination power of several parameters has been evaluated for adult and paediatric arrhythmias (Aramendi et al., 2006; 2010; 2007; Irusta et al., 2008; Ruiz de Gauna et al., 2008). These studies underlined the inadequacy of many parameters and discrimination thresholds for discriminating VT from SVT safely when adult and paediatric rhythms were considered. A reliable SVT/VT discrimination algorithm is therefore a key requirement for adapting or developing SAA for paediatric use.

This chapter comprises five sections. Section 2 describes the database compiled to develop and test a SVT/VT discrimination algorithm in the context of AED arrhythmia classification. More than 1900 records from adult and paediatric patients were compiled, and a thorough description of the sources and rhythm classification process is given. Special attention is paid to the SVT/VT subset, which contains more than 650 records. The criteria for classification are reported, and heart rate analysis for both adult and paediatric populations is detailed. In section 3, we conduct the spectral analysis of SVT and VT rhythms and define a number of spectral parameters that discriminate between VT and SVT. Emphasis is placed on the influence of the age group and the heart rate on the values of the parameters. Based on these spectral parameters, a SVT/VT discrimination algorithm is designed in section 4 . The discriminative power of the algorithm is assessed through the receiver operating characteristic (ROC) curve and the sensitivity/specificity values. The algorithm accurately discriminates between SVT and VT in both adults and children, and it could safely be incorporated into

\footnotetext{
${ }^{1}$ The latest version of the guidelines was released in 2010 (Biarent et al., 2010).
} 
SAA. Section 5 discusses the main conclusions of this work and analyzes what issues need to be addressed when using adult SAA with children. Several strategies to adapt SAA for paediatric use are discussed, and an overall scheme to adapt adult SAA for paediatric use, integrating the SVT/VT discrimination algorithm described in section 4, is proposed.

\section{Adult and paediatric data collection}

The accurate identification of VT and SVT by an AED is well described in the 1997 AHA statement on the performance and safety of AED SAA (Kerber et al., 1997). The AHA statement describes the composition of the databases used to develop and test SAA, including the types of rhythms and the minimum number of records per rhythm type. It divides the rhythm types into three categories: shockable, nonshockable and intermediate ${ }^{2}$. It also specifies that within a rhythm type, all records must be obtained from different patients. Finally, the statement defines the performance goals of the SAA for shockable and non-shockable rhythms, i.e. the minimum sensitivity and specificity of the SAA per rhythm type. These values are exhibited in table 1.

\begin{tabular}{|c|c|c|c|c|}
\hline Rhythms & $\begin{array}{l}\text { Minimun no. of } \\
\text { records }\end{array}$ & Performance goal & $\begin{array}{c}\text { Observed } \\
\text { performance }\end{array}$ & $90 \%$ One-sided CI \\
\hline \multicolumn{5}{|l|}{ Shockable } \\
\hline Coarse VF ${ }^{a}$ & 200 & $>90 \%$ Sens & $>90 \%$ & $87 \%$ \\
\hline Rapid VT & 50 & $>75 \%$ Sens & $>75 \%$ & $67 \%$ \\
\hline Nonshockable & 300 total & & & \\
\hline NSR & 100 & $>99 \%$ Spec & $>99 \%$ & $97 \%$ \\
\hline Other & 30 & $>95 \%$ Spec & $>95 \%$ & $88 \%$ \\
\hline Asystole & 100 & $>95 \%$ Spec & $>95 \%$ & $92 \%$ \\
\hline \multicolumn{5}{|l|}{ Intermediate } \\
\hline Fine VF & 25 & Report only & - & - \\
\hline Other VT & 25 & Report only & - & - \\
\hline
\end{tabular}

a Peak-to-peak amplitude above $200 \mu \mathrm{V}$.

b Specified by the manufacturer, because tolerance to VT varies widely among patients.

Table 1. Definition of the databases used to validate SAA, adapted from the AHA statement (Kerber et al., 1997). The other nonshockable rhythms include supraventricular tachycardia (SVT), atrial fibrillation, premature ventricular contractions, heart blocks, sinus bradycardia and idioventricular rhythms. The $90 \%$ one-sided confidence intervals (CI) are computed for the observed performance goals. no.=number; NSR=normal sinus rhythm; Sens=sensitivity; $\mathrm{Spec}=$ specificity; $\mathrm{VF}=$ ventricular fibrillation; $\mathrm{VT}=$ =ventricular tachycardia.

Currently, there exists no public database of ECG records compliant with the AHA statement. Each AED manufacturer compiles its own data, which must include paediatric rhythms if the AED will be used to treat children. However, the studies describing paediatric databases (Atkins et al., 2008; Atkinson et al., 2003; Cecchin et al., 2001) report fewer shockable rhythms than those specified in the AHA statement because paediatric ventricular arrhythmias are scarce.

\footnotetext{
${ }^{2}$ Rhythms for which the benefits of defibrillation are uncertain.
} 
An adult and a paediatric database were compiled using ECG records collected from in- and out-of-hospital sources. Three cardiologists assigned a rhythm type and a shock/no-shock recommendation to each record. For potentially shockable rhythms, the criteria by which to determine the shock/no-shock recommendation were: the patient was unresponsive, had no palpable pulse, and was of unknown age (Cecchin et al., 2001). Diagnostic discrepancies among the reviewers were further discussed, and a consensus decision for the shock/no-shock recommendation was agreed upon after the assessment of the risks. Most of the reviewer disagreements in diagnosis occurred between SVT and VT rhythms.

The database contains shockable rhythms (VF and rapid VT) and the most representative nonshockable rhythms: normal sinus rhythm (NSR) and SVT. Although the AHA includes SVT among the other nonshockable rhythms (see table 1), we have added an individual SVT category because adult SAA may fail to detect high-rate paediatric SVT (Atkins et al., 2008) accurately. Although the complete database is initially described, the SVT/VT subset was extracted to study VT discrimination. We considered VT shockable for heart rates above $150 \mathrm{bpm}$ in adults and $20 \mathrm{bpm}$ above the age-matched normal rate in children (Atkinson et al., 2003), which amounts to $180 \mathrm{bpm}$ in infants (under 1 year) and $150 \mathrm{bpm}$ in children older than 1 year. Table 2 presents a summary of the database, where the paediatric data are divided into three age groups: under 1 year, $1-8$ years (ILCOR recommendation) and above 8 years. All records were stored with a common format and sampling frequency of $f_{s}=250 \mathrm{~Hz}$.

\begin{tabular}{|c|c|c|c|c|c|c|}
\hline \multirow[b]{2}{*}{ Rhythms } & \multicolumn{4}{|c|}{ Paediatric } & \multirow[b]{2}{*}{ Adult } & \multirow[b]{2}{*}{ Minimum $^{a}$} \\
\hline & $<1 \mathrm{y}$ & $1 y-8 y$ & $>8 y$ & Total & & \\
\hline \multicolumn{7}{|l|}{ Shockable } \\
\hline Coarse VF & $3(1)$ & $18(11)$ & $37(10)$ & $58(22)$ & $374(374)$ & 200 \\
\hline Rapid VT & $8(4)$ & 39 (19) & $19(13)$ & $66(33)$ & $200(200)$ & 50 \\
\hline \multicolumn{7}{|c|}{ Nonshockable } \\
\hline NSR & $14(13)$ & $312(280)$ & 214 (161) & $540(454)$ & $292(292)$ & 100 \\
\hline SVT & $38(29)$ & 147 (103) & 137 (104) & $322(236)$ & 89 (89) & 30 \\
\hline Total & $63(39)$ & $516(357)$ & 407 (216) & $986(612)$ & $955(820)$ & - \\
\hline
\end{tabular}

a As specified in the AHA statement for adult records, see table 1.

Table 2. Number of records per rhythm class in the adult and paediatric databases. The number of patients is indicated in parentheses. Asystole and intermediate rhythms were excluded from the analysis, and the others category is composed of SVT. The SVT/VT subset is highlighted.

\subsection{Adult records}

The adult database contains 955 records from 820 patients: 574 shockable records from 541 patients and 381 nonshockable records from 351 patients. The mean duration of the records was $13.0 \pm 5.3 \mathrm{~s}$ overall, with $15.4 \pm 4.2 \mathrm{~s}$ for the nonshockable and $11.4 \pm 5.4 \mathrm{~s}$ for the shockable records. The adult database was compiled following the AHA statement; hence, all records within a rhythm type come from different patients. 
The adult records were obtained from three sources. Two hundred fifty-one nonshockable and 63 shockable records were extracted from public ECG databases ${ }^{3}$. The adult data also include 127 nonshockable and 325 shockable records from in-hospital electrophysiology (EP) studies and intensive care units at two Spanish hospitals (Basurto and Donostia hospitals). Finally, the database contains three nonshockable and 186 shockable out-of-hospital records recorded by two Spanish emergency services in Madrid and in the Basque Country.

Public databases are available in digital format with different sampling rates and storage formats. In-hospital data were gathered in digital format (Prucka Cardiolab and EP-Tracer systems) or as printed ECG paper strips. All out-of-hospital data came in paper format from AED printouts.

\subsection{Paediatric records}

The paediatric database contains 986 records from 612 paediatric and adolescent patients aged between 1 day and 20 years (mean age, $7.1 \pm 4.5$ years). There are 862 nonshockable records from 579 patients and 124 shockable records from 49 patients. The mean duration of the records was $13.7 \pm 9.0 \mathrm{~s}$, with $14.1 \pm 9.3 \mathrm{~s}$ for the nonshockable and $10.9 \pm 4.9 \mathrm{~s}$ for the shockable records.

Shockable paediatric rhythms were difficult to obtain because lethal ventricular arrhythmias are rare in children. Furthermore, their occurrence increases with age, and this is reflected in our database, in which most VF rhythms were collected from patients aged 9 years or older. Several records from the same patient were included in the same rhythm type when the morphology of the arrhythmias was different. This procedure is contrary to the AHA statement; however, all studies on the use of SAA in children have allowed rhythm repetition because paediatric ventricular arrhythmias are scarce (Atkins et al., 2008; Atkinson et al., 2003; Cecchin et al., 2001; Irusta \& Ruiz, 2009).

All the paediatric records were collected in hospitals, from archived paper and digital EP studies (Prucka Cardiolab and EP-Tracer systems). The records were retrospectively obtained from five Spanish hospitals: Cruces, Donostia, La Paz, Gregorio Marañón and San Joan de Deu.

\subsection{Analysis of the SVT/VT subset}

Accurate discrimination between SVT and VT is crucial in order to adapt adult SAA for paediatric use (Atkins et al., 2008; Irusta \& Ruiz, 2009). Consequently, the SVT/VT subset was extracted from the collected data in order to analyze the accurate detection of VT in the context of a SAA. We will use this subset to compare the characteristic features of each rhythm and to define a set of parameters that discriminate between SVT and VT in adult and paediatric patients.

The SVT/VT database consists of 677 rhythms distributed as described in table 2. There are 89 adult and 322 paediatric SVT records, and 200 adult and 66 paediatric VT records. The subset includes a large number of paediatric SVT, which is, in the context of SAA, the most challenging rhythm type for accurate detection of VT (Aramendi et al., 2010; Atkins et al., 2008; Irusta \& Ruiz, 2009).

\footnotetext{
$\overline{{ }^{3} \text { The MIT-BIH }}$ arrhythmia, the AHA and Creighton University ventricular tachyarrhythmia databases.
} 
Several records from the SVT/VT database were the most difficult to agree on for the cardiologists who classified the records in our databases. Based on a single-lead recording of a duration of approximately $10 \mathrm{~s}$, consensus was not always easy. Fig. 1 shows four paediatric examples in which similar heart rates correspond to different rhythms. The cases shown in Figs 1(a) and 1(d) have the same $255 \mathrm{bpm}$ heart rate, but there is atrial activity in the former and exclusively ventricular activity the latter. In general, disagreements were resolved by adopting the original interpretation of the physician aware of the clinical history of the patient. This interpretation is more reliable, but it is only available when records are obtained from documented EP studies. For out-of-hospital records, forced consensus was used to integrate the record in the database.

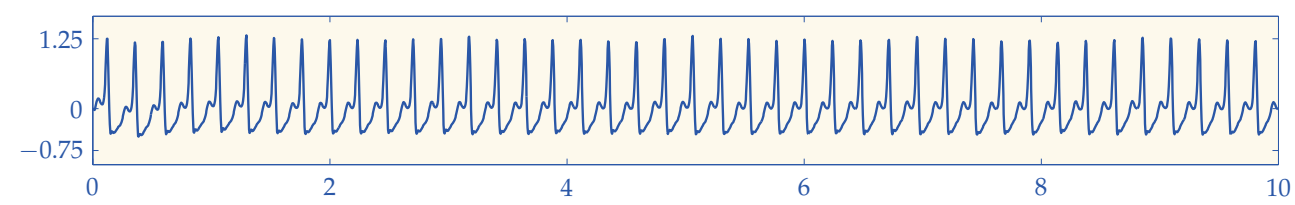

(a) Paediatric SVT with disagreements in diagnosis (heart rate $255 \mathrm{bpm}$ ).

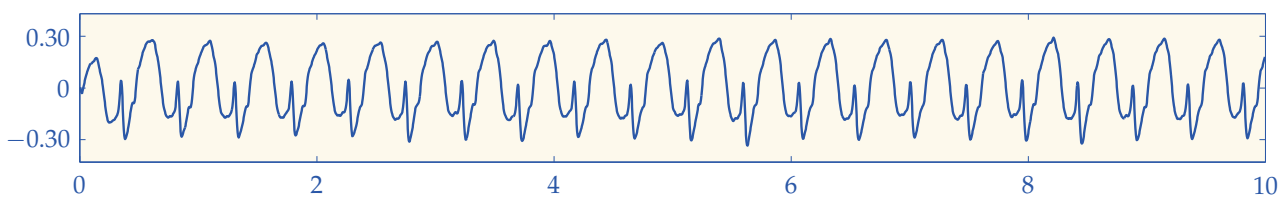

(b) Paediatric SVT with disagreements in diagnosis (heart rate $125 \mathrm{bpm}$ ).

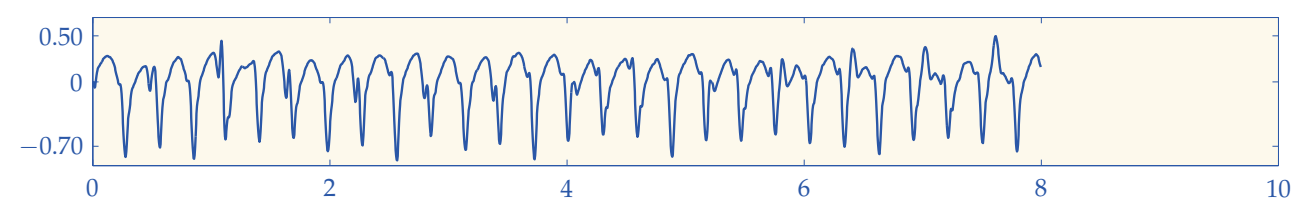

(c) Paediatric VT with disagreements in diagnosis (heart rate $210 \mathrm{bpm}$ ).

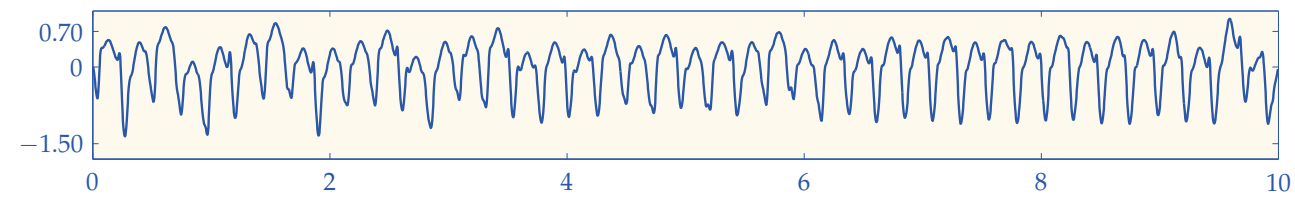

(d) Paediatric VT with disagreements in diagnosis (heart rate $255 \mathrm{bpm}$ ).

Fig. 1. Paediatric supraventricular tachycardia (SVT) and ventricular tachycardia(VT) records that required a forced consensus between cardiologists for the rhythm classification.

For an accurate SVT/VT diagnosis, attention should be paid to the differences in the ECG between adults and children. It is well known that to maintain cardiac output, children present higher heart rates to compensate for smaller stroke volumes (Rustwick et al., 2007). With age, stroke volume increases and contributes more significantly to overall cardiac output. The duration of the QRS complex is shorter in children than in adults because of the smaller 
cardiac muscle mass in children (Chan et al., 2008), while the QT interval is similar in children and adults. The QT interval, however, depends on and is normally adjusted to the heart rate.

SVT is the most common paediatric tachydysrhythmia. SVT heart rates are typically above $220 \mathrm{bpm}$ in infants and above $180 \mathrm{bpm}$ in children (Manole \& Saladino, 2007). SVT is usually associated with an accessory atrioventricular pathway in neonates and young children. In adolescents and adults, the most common cause of SVT is an atrioventricular nodal reentry tachycardia (Chan et al., 2008).

Although SVT is more common in children, tachycardias of ventricular origin do occur in paediatric patients (Chan et al., 2008). As the normal QRS complex is of shorter duration in children than in adults, VT is more difficult to diagnose in young children. What appears as a slightly prolonged QRS complex in the ECG may, in fact, represent a significantly prolonged or wide complex tachycardia in infants and children. Consequently morphology features rather than rate information should be considered by the SVT/VT discrimination algorithm.

\subsection{Analysis of the heart rate}

Heart rate (HR) calculations are inherent to many SAA because fatal ventricular arrhythmias are associated with very high ventricular rates. We have analyzed the HR for the SVT/VT database in order to assess the effect of patient age on the HR for SVT and VT rhythms. QRS complexes were automatically detected, and results were visually inspected and corrected when necessary. The value of the HR for each record was computed as the inverse of the mean $R R$ interval, and the result is given in beats per minute (bpm) ${ }^{4}$ :

$$
\mathrm{HR}(\mathrm{bpm})=60 \cdot \frac{1}{\overline{R R}(s)},
$$

where $\overline{R R}$ is the mean $R R$ interval expressed in seconds.

Table 3 shows the mean HR of the nonshockable SVT and the shockable VT records for all age groups-adult and paediatric. The mean values of the HR were compared using the unequal variance t-test and a value of $p<0.001$ was considered significant. The HR for paediatric SVT (187 bpm) was significantly higher than for adult SVT $(131 \mathrm{bpm})(p<0.001)$. Furthermore, the database contains 19 SVT values from infants with HR values above $180 \mathrm{bpm}$ and 222 SVT values from children aged 1 year or older with HR values above $150 \mathrm{bpm}$; i.e., with rates above the threshold for shockable VT. The mean HR values did not differ significantly between adult VT $(241 \mathrm{bpm})$ and paediatric VT $(232 \mathrm{bpm})(p=0.39)$.

The high rate of paediatric SVT largely overlaps with paediatric and adult VT rates. This fact is clearly shown in Fig. 2, in which the normalized histograms of the HR values for the SVT and VT records are plotted for the different populations. The overlap between the HR histograms of SVT and VT records is larger in the paediatric population (Fig. 2(b)) than in the adult population (Fig. 2(a)). When both population groups are aggregated (Fig. 2(c)), the overlap remains because the HR of paediatric SVT overlaps with that of adult and paediatric VT. This overlap precludes poor SVT/VT discrimination based exclusively on the HR.

\footnotetext{
${ }^{4}$ All the signal processing algorithms and calculations described in this chapter were carried out using MATLAB (MathWorks, Natick, MA), version 7.10.0.
} 


\begin{tabular}{lccccccc}
\hline \multirow{2}{*}{ Rhythms } & \multicolumn{9}{c}{ Paediatric } & & \\
\cline { 2 - 5 } & $<1 \mathrm{y}$ & $1 \mathrm{y}-8 \mathrm{y}$ & $>8 \mathrm{y}$ & Total & & Adult & $p$ value \\
\hline SVT & $186 \pm 45$ & $194 \pm 39$ & $180 \pm 39$ & $187 \pm 40$ & $131 \pm 32$ & $<0.001$ \\
VT & $226 \pm 31$ & $247 \pm 57$ & $206 \pm 46$ & $232 \pm 54$ & $241 \pm 58$ & 0.3857 \\
\hline
\end{tabular}

Table 3. Mean heart rate (HR) (mean \pm standard deviation) expressed in bpm for the SVT/VT database. The difference in mean HR between adult and paediatric patients is significant for SVT but not for VT.

Fig. 3 shows the results of the analysis of the HR for the complete database, including NSR, VT and SVT rhythms ${ }^{5}$. The HR distribution shows a clearer separation between VT and the nonshockable rhythms (NSR and SVT), particularly for the adult patients (Fig. 3(a)). Although a SAA based on HR dependent parameters might be reliable enough for adults, such an approach will fail when high-rate paediatric SVT is considered, compromising both paediatric SVT specificity and the overall VT sensitivity.

Many SAAs base their shock/no-shock decisions on heart rate calculations. Discrimination parameters of adult SAA that strongly depend on the heart rate fail to diagnose paediatric SVT accurately as nonshockable (Aramendi et al., 2010; Atkins et al., 2008), compromising the specificity of the SAA. The addition of high-rate paediatric SVT to a database to test SAA may compromise not only SVT specificity, but also VT sensitivity. It is therefore necessary to develop safe SVT/VT discrimination algorithms based on heart rate independent features.

\section{Spectral analysis}

As shown in Fig. 2, the HR of paediatric SVT presents a large overlap with the HR of adult and paediatric VT. The accurate discrimination of VT by an AED SAA must therefore rely on ECG features not affected by the HR. Power spectral analysis of the ECG, which quantifies the power distribution of the ECG as a function of frequency, is an adequate and simple tool for the definition of HR-independent features.

\subsection{Power spectral distribution}

Following standard AED practice, the ECG was first preprocessed with an order 6 Butterworth band-pass filter $(0.7-35 \mathrm{~Hz})$ to suppress baseline wander and power line interferences. The preprocessed ECG, $x_{\mathrm{ecg}}$, was analyzed using nonoverlapping segments of $3.2 \mathrm{~s}$ duration, and a maximum of three segments per record were used. Each segment was windowed using a Hamming window, and the power spectral density (PSD) of the segment was estimated as the square of the amplitude of the fast Fourier transform (FFT), zero padded to 4096 points.

Some frequency components of the ECG segment were made zero: frequency components outside the $0.7-35 \mathrm{~Hz}$ analysis band; and insignificant components, defined as those with amplitudes below $5 \%$ of the maximum amplitude of the FFT (Barro et al., 1989). The PSD

\footnotetext{
${ }^{5}$ VF records were excluded from the analysis because VF is an irregular ventricular rhythm characterized by the absence of QRS complexes and a well-defined heart rate.
} 


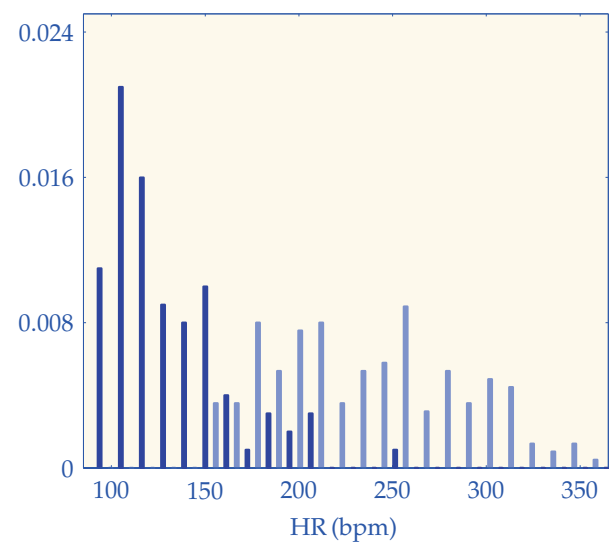

(a) HR distribution of the adult SVT and VT records.

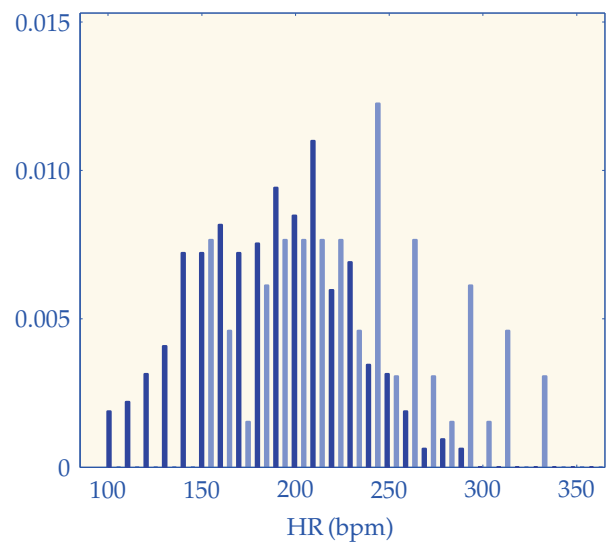

(b) HR distribution of the paediatric SVT and VT records.

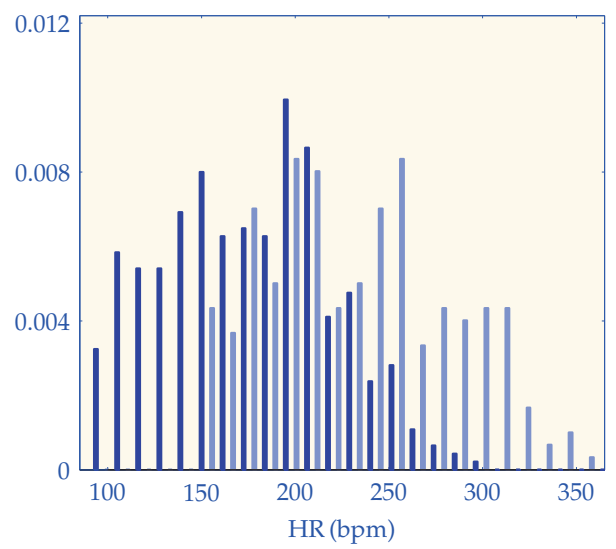

(c) HR distribution of SVT and VT records, for the adult and paediatric populations.

Fig. 2. Heart rate (HR) distributions for each population group for the SVT records ( $\square$ ) and VT records $(\square)$.

normalized to a unit area under the curve, $P_{x x}(f)$, was then estimated as:

$$
P_{x x}(f)=\frac{\left|X_{\mathrm{ecg}}(f)\right|^{2}}{\sum_{f=0.7 \mathrm{~Hz}}^{35 \mathrm{~Hz}}\left|X_{\mathrm{ecg}}(f)\right|^{2}},
$$

where $X_{\mathrm{ecg}}(f)$ is the FFT of the $3.2 \mathrm{~s}$ segment after zeroing the components mentioned above. $P_{x x}(f)$ was then used to define a set of spectral features that capture the morphological differences between the PSD of VT and SVT rhythms. 


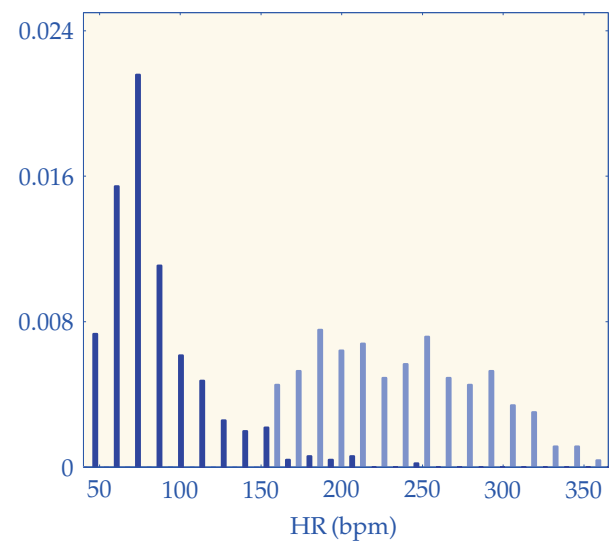

(a) HR distribution of the adult records in the complete database.

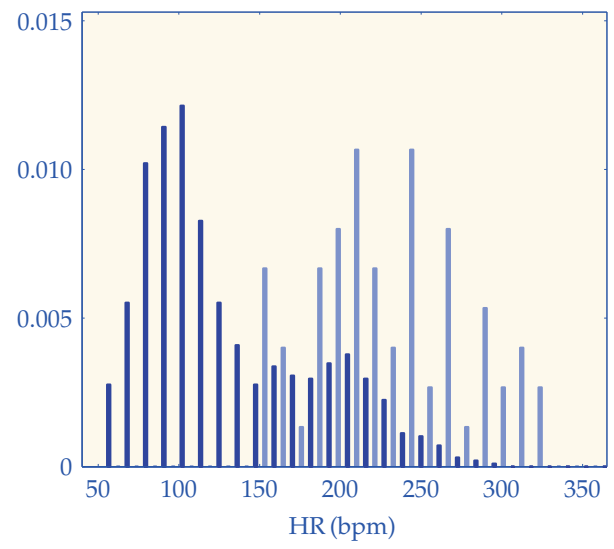

(b) HR distribution of the paediatric records in the complete database.

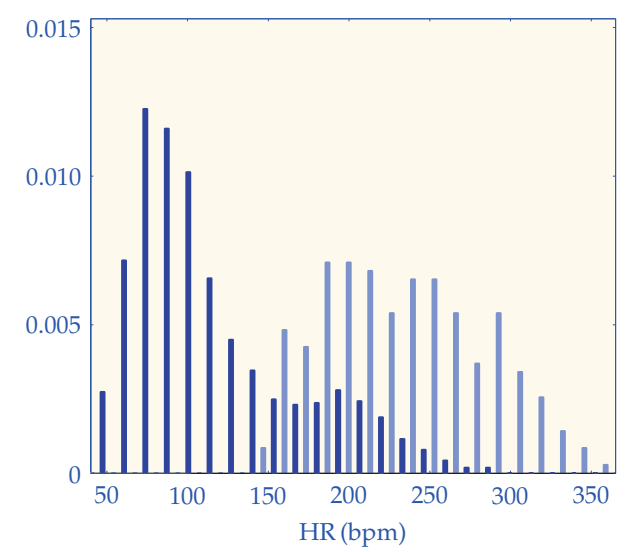

(c) HR distribution of the records in the complete database, adult and paediatric combined.

Fig. 3. HR distributions for each population group for the complete database: nonshockable records $(\square)$ and VT records ( $\square)$.

Both monomorphic VT and SVT are regular rhythms. The morphology of the QRS complex changes slowly from beat to beat, and beats occur at very regular intervals. These rhythms can therefore be regarded as quasiperiodic, and their power spectrum concentrates around the harmonics of the fundamental frequency. The beat repetition period is the mean $R R$ interval, $\overline{R R}$, so the fundamental frequency is the cardiac frequency:

$$
f_{c}(H z)=\frac{1}{\overline{R R}(s)} .
$$

The harmonics are located at the integer multiples of the cardiac frequency:

$$
f_{k}=k \cdot f_{c} .
$$


Although SVT and VT distribute their power around the harmonic frequencies, $f_{k}$, the relative power content of each harmonic is very different in SVT and VT. The examples in Fig. 4, which represent a typical $P_{x x}(f)$ for a VT and a SVT, illustrate such differences.

Monomorphic VT presents wide QRS complexes that frequently resemble a sinus-like waveform. In the frequency domain, most of the power of the ECG is concentrated in a narrow frequency band around $f_{c}$, which corresponds to the ventricular rate of the VT (see Fig. 4(a) for an example). Rather than using the $R R$ intervals computed in the time domain, the ventricular rate can easily be estimated in the frequency domain as the frequency at which $P_{x x}(f)$ is at its maximum:

$$
f_{d}(H z)=\underset{f \in(0.7-35)}{\arg \max }\left\{P_{x x}(f)\right\} .
$$

This frequency is called the dominant frequency and has been extensively used to characterize ventricular arrhythmias and to estimate the effectiveness of defibrillation shocks (Strohmenger et al., 1996).
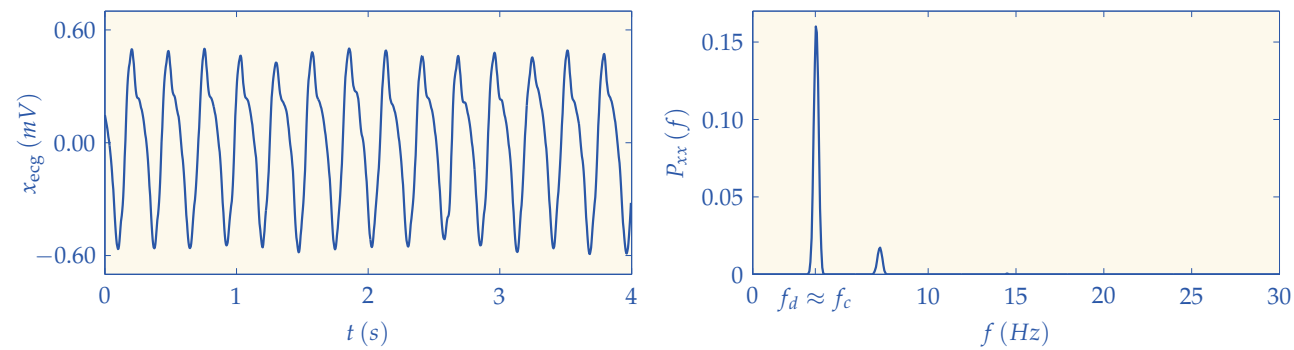

(a) A VT with a ventricular rate of $214 \mathrm{bpm}(3.6 \mathrm{~Hz})$ in the time and frequency domains. For VT most of the power is concentrated around the dominant frequency, $f_{d} \approx f_{c}=4 \mathrm{~Hz}$, which corresponds to the ventricular rate.

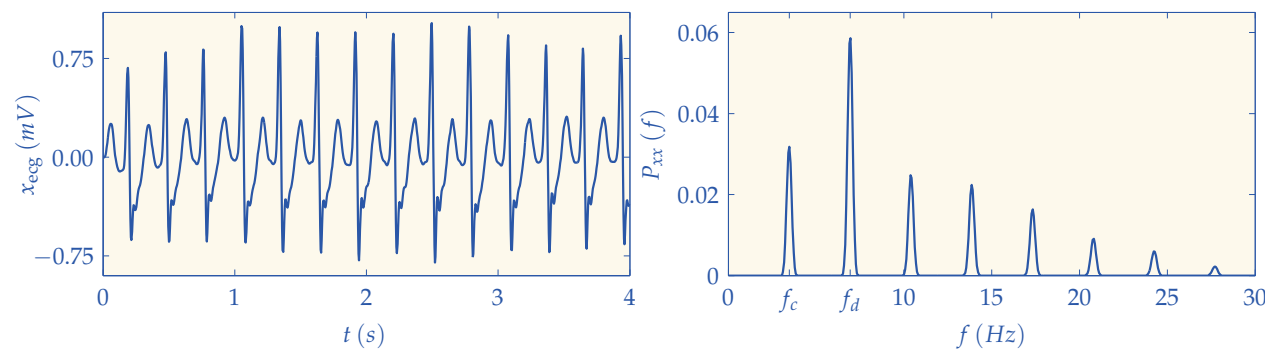

(b) A SVT with a heart rate of $208 \mathrm{bpm}(3.5 \mathrm{~Hz})$ in the time and frequency domains. In this case, the dominant frequency appears in the second harmonic, $f_{d} \approx 2 f_{c}=7 \mathrm{~Hz}$, which corresponds to twice the heart rate. Most of the power is distributed among the higher harmonics.

Fig. 4. Examples of a SVT and VT in the time and frequency domains. Although in both cases power is distributed among the harmonics of $f_{c}$, the power content of the harmonics is very different.

During SVT, the ECG changes more abruptly; QRS complexes are narrower, and the signal power is distributed among several harmonics of the cardiac frequency. The number of harmonics can be large because of the rapid variations in the QRS complexes, and the power 


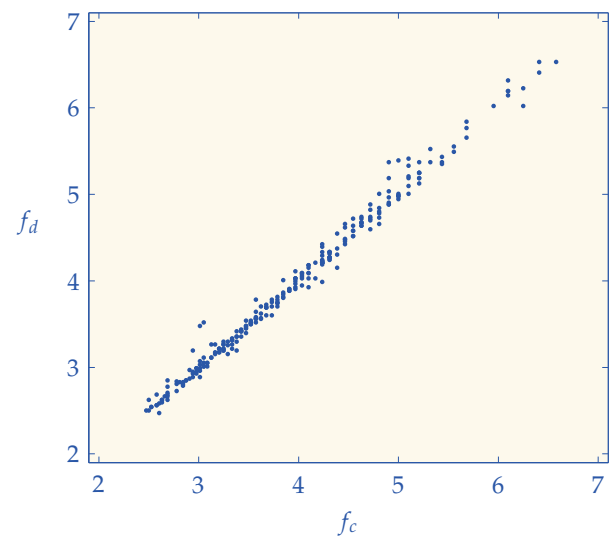

(a) VT. The dominant frequency accurately represents the ventricular rate.

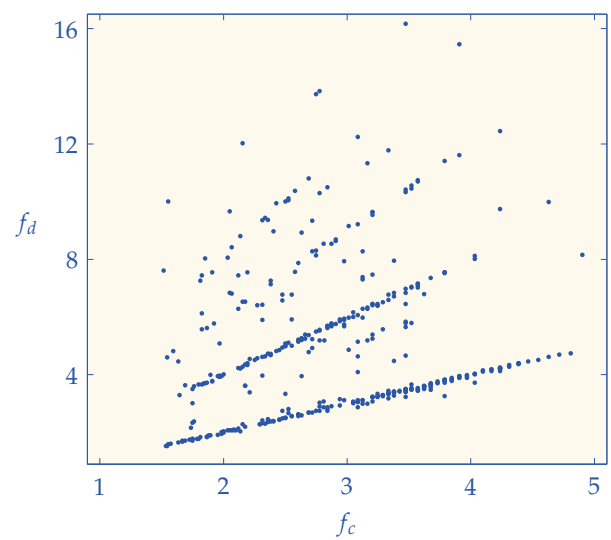

(b) SVT. The dominant frequency frequently appears in higher harmonics as shown by lines of increasing slope.

Fig. 5. Relation between $f_{d}$ computed in the frequency domain and $f_{c}$ computed in the time domain for SVT and VT.

is therefore distributed in a larger frequency band than for VT, as shown in the example in Fig. 4(b). Frequently, $P_{x x}(f)$ is at its maximum at a high harmonic $(k>1)$, so the dominant frequency is a multiple of the cardiac frequency rather than the cardiac frequency itself $(k=1)$.

\subsection{Relation between $f_{d}$ and $f_{c}$ in SVT and VT}

We conducted a standard Pearson correlation analysis in order to investigate further the relation between $f_{c}$ and $f_{d}$ in our database of SVT and VT. We used the first $3.2 \mathrm{~s}$ segment to estimate $f_{d}$. In this way, we compared the cardiac frequency obtained from the identification of the QRS complexes in the time domain $f_{c}$, equation (3), to the dominant frequency obtained from the spectral analysis of the ECG, $f_{d}$.

Fig. 5 shows the relation between $f_{d}$ and $f_{c}$ for all the SVT and the VT records in the database. As shown in Fig. 5(a), $f_{d} \approx f_{c}$ for all VT instances in our database; there are no outliers, and the correlation coefficient is almost one $(r=0.998)$. The relation is more complex for SVT, however, as shown in Fig. 5(b). There are four lines of increasing slope corresponding to $f_{d}=k \cdot f_{c}$ for $k=1, . ., 4$, and the correlation coefficient is low $(r=0.134)$. Furthermore, these lines cover cardiac frequencies in the $2-4 \mathrm{~Hz}$ range, indicating that the dominant frequency may fall in a higher harmonic for a large range of SVT heart rates (120-240 bpm). When the cardiac frequency of SVT is above $4 \mathrm{~Hz}$, the dominant frequency corresponds to the cardiac frequency.

The mean values for $f_{d}, f_{c}$ and the correlation coefficient between these two variables $r\left(f_{d}, f_{c}\right)$ are compiled in Table 4 . This table presents the results for both types of rhythms and also for adult and paediatric patients. The differences in the values of the frequencies between adult and paediatric rhythms are not significant for VT, while paediatric SVT presents significantly higher dominant and cardiac frequencies, as expected from the HR analysis described in section 2.3. The relative difference between the dominant and the cardiac frequency is smaller 
in the paediatric population because paediatric SVT with heart rates above $240 \mathrm{bpm}$ are frequent in our database, and in those cases, $f_{d} \approx f_{c}$.

In summary, the dominant frequency of VT rhythms has a clear physiological interpretation as the ventricular rate of the arrhythmia, which can therefore be easily estimated in the frequency domain. For SVT, the dominant frequency customarily falls in one of the first four harmonic frequencies and only when the heart rate is very high (above $240 \mathrm{bpm}$ ) can it be interpreted as the heart rate.

\subsection{Distribution of the power content}

In this section we analyze how the power of the ECG is distributed among the different harmonics of the cardiac frequency. VT rhythms will concentrate most of their power around the fundamental component $\left(f_{c} \approx f_{d}\right)$. For SVT, the signal power will be distributed among several harmonics of $f_{c}$. These differences are caused by the morphology of the arrhythmias-wide sinus-like QRS complexes in VT and narrower QRS complexes in SVT-and are, for the most part, independent of the heart rate.

Let $P_{k}$ stand for the power content percentage of the $k^{\text {th }}$ harmonic. We estimated this value by adding all power components in a $f_{c}(\mathrm{~Hz})$ frequency band centered in $k \cdot f_{c}$ :

$$
P_{k}(\%)=100 \cdot \sum_{k f_{c}-0.5 f_{c}}^{k f_{c}+0.5 f_{c}} P_{x x}(f) \quad k=1, \ldots, 10 .
$$

A graphical example of the computation of the power of the harmonics is shown in Fig. 6 for a SVT and a VT.

Table 5 compares the values of $P_{k}$ between SVT and VT for all age groups in our database: paediatric, adult and all patients. VT rhythms present small differences in harmonic content between adults and children. On average, $P_{1}$ accounts for over $80 \%$ of the total power, and it always has the largest power content-as we already know from the analysis of the dominant frequency. The value of $P_{1}$ slightly increases as the heart rate increases, but the dependence is very weak $(r=0.28)$, as shown in Fig. 7. Although the difference is not large, $P_{1}$ is smaller in paediatric VT than in adult VT; in fact, children may have VT with narrower QRS complexes with durations under $90 \mathrm{~ms}$ (Schwartz et al., 2002). The total power content of the first three harmonics accounts for over $95 \%$ of the total power in VT, both in children and in adults.

\begin{tabular}{ccccccccc}
\hline & \multicolumn{2}{c}{ paediatric } & & \multicolumn{2}{c}{ Adult } & & \multicolumn{2}{c}{ Total } \\
\cline { 2 - 3 } Parameter & SVT & VT & & SVT & VT & & SVT & VT \\
\hline$f_{c}(\mathrm{~Hz})$ & $3.1 \pm 0.7$ & $3.9 \pm 0.9$ & & $2.2 \pm 0.5$ & $4.0 \pm 1.0$ & & $2.9 \pm 0.8$ & $4.0 \pm 1.0$ \\
$f_{d}(\mathrm{~Hz})$ & $5.2 \pm 2.8$ & $3.9 \pm 0.9$ & & $4.3 \pm 2.6$ & $4.0 \pm 1.0$ & & $5.0 \pm 2.8$ & $4.0 \pm 1.0$ \\
$r\left(f_{d}, f_{c}\right)$ & 0.06 & 1.00 & & 0.19 & 1.00 & & 0.13 & 1.00 \\
& $(-0.05-0.17)$ & $(1.00-1.00)$ & & $(-0.02-0.38)$ & $(1.00-1.00)$ & & $(0.04-0.23)$ & $(1.00-1.00)$ \\
\hline
\end{tabular}

Table 4. Mean value (mean \pm standard deviation) of $f_{c}$ and $f_{d}$ by rhythm type and population group. The correlation coefficient $r\left(f_{d}, f_{c}\right)$ between these variables and its corresponding $95 \% \mathrm{CI}$ are also reported. 
On average, SVT presents up to six significant harmonics ${ }^{6}$, although some adult SVT have over 10 significant harmonics. When the heart rate is low, SVT may present a larger number of significant harmonics. This explains why the power content of the lower harmonics is smaller in adult than in paediatric SVT. On average, the higher SVT harmonics $(k \geq 4)$ contain $40 \%$ of the total power: $47 \%$ in adults and $37 \%$ in children. In this study, we define $P_{H}$, the

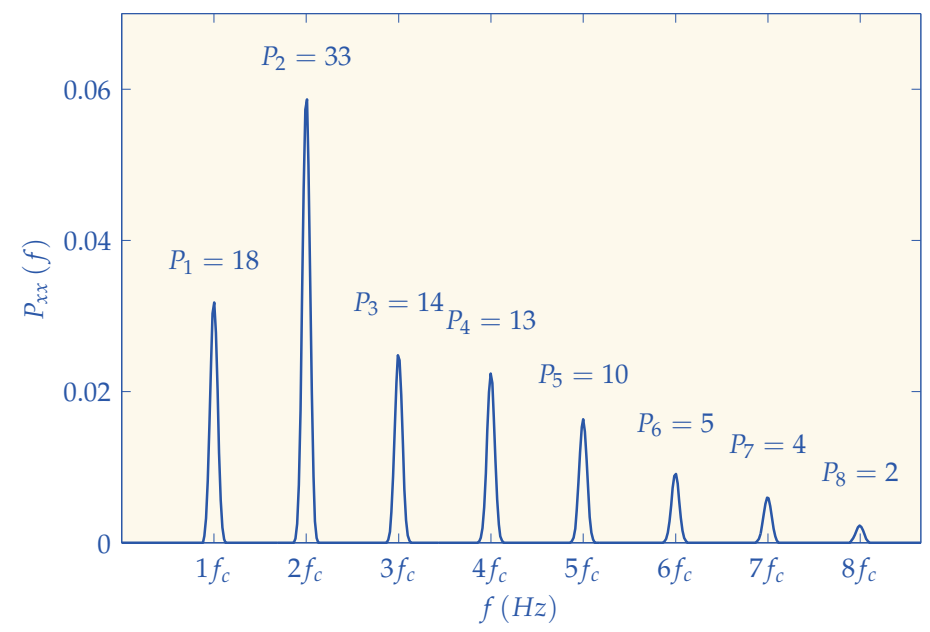

(a) Power is distributed among several harmonics in SVT, with up to six significant harmonics.

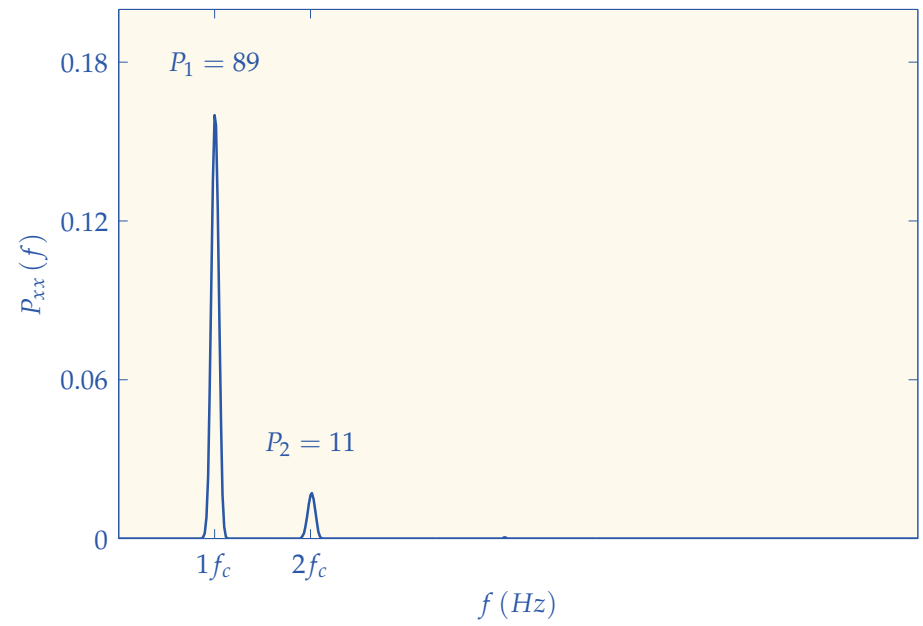

(b) Most power is concentrated around the fundamental component in VT $\left(f_{c}=f_{d}\right)$.

Fig. 6. Computation of the power content of the harmonics, for the examples shown in Fig.4.

\footnotetext{
${ }^{6}$ Harmonics that contribute at least $5 \%$ of the total power.
} 


\begin{tabular}{|c|c|c|c|c|c|c|}
\hline \multirow[b]{2}{*}{$P_{k}$} & \multicolumn{2}{|c|}{ paediatric } & \multicolumn{2}{|c|}{ Adult } & \multicolumn{2}{|c|}{ Total } \\
\hline & SVT & VT & SVT & VT & SVT & VT \\
\hline$k=1$ & $28(6-56)$ & $83(71-92)$ & $23(3-51)$ & $88(76-96)$ & $27(5-55)$ & $86(75-96)$ \\
\hline$k=2$ & $19(8-31)$ & $10(3-18)$ & $18(7-33)$ & $8(2-17)$ & $19(8-32)$ & $9(2-18)$ \\
\hline$k=3$ & $16(8-23)$ & $3(1-8)$ & $12(6-19)$ & $2(0-4)$ & $15(7-22)$ & $2(0-6)$ \\
\hline$k=4$ & $12(6-17)$ & $2(0-4)$ & $11(5-17)$ & $1(0-2)$ & $12(6-17)$ & $1(0-3)$ \\
\hline$k=5$ & $8(3-13)$ & $1(0-2)$ & $9(4-12)$ & $0(0-1)$ & $9(3-13)$ & $0(0-1)$ \\
\hline$k \geq 6$ & $17(3-36)$ & $1(0-1)$ & $25(5-48)$ & $0(0-1)$ & $18(3-40)$ & $0(0-1)$ \\
\hline
\end{tabular}

Table 5. Mean content of the harmonics by rhythm type and population group. The range in parentheses represents the 10-90 percentile range.

power content of the higher harmonics $(k \geq 4)$, in the following way:

$$
P_{H}=\sum_{k \geq 4} P_{k}
$$

The values of $P_{H}$ slowly decrease as the heart rate increases, as shown in Fig. 8, which explains the differences in power distribution between adult and paediatric SVT. These differences are not large, however, because $P_{H}$ depends very weakly on the heart rate $(r=-0.37)$.

In summary, the differences in spectral content between SVT and VT are large, regardless of the age group. VT concentrates its power around the fundamental component: on average, $P_{1}$ contains over $80 \%$ of the total power, and $P_{H}$ less than $5 \%$. The small differences between adult and paediatric VT are caused by the narrower QRS complexes in paediatric VT, since the heart rates of adult and paediatric VT in our database are not significantly different. SVT distributes its power among many harmonics: on average, $P_{1}$ contains less than $30 \%$ of the total power, and $P_{H}$ more than $35 \%$. The power content of the higher harmonics is smaller in paediatric SVT than in adult SVT because the heart rate in paediatric SVT is larger; however, the differences are not large.

\section{An accurate SVT/VT discrimination algorithm}

The spectral differences between SVT and VT described in the previous section are, for the most part, independent of the heart rate. An accurate SVT/VT discrimination algorithm valid for adult and paediatric patients can therefore be designed based on those differences. In this work, we propose the use of two spectral features described in the preceding section: $P_{1}$ and $P_{H}$.

Fig. 9(a) and Fig. 9(b) show the histograms of the two spectral parameters for the SVT and VT records, both in adult and paediatric cases. Both parameters show a clear separation, which is independent of the age group, between the types of arrhythmias. This confirms their adequacy for discriminating between VT and SVT accurately in children and adults.

\subsection{Classification algorithm}

The adult and paediatric databases were randomly split into two groups containing equal numbers of patients. The first database was used to develop the SVT/VT algorithm, and the 


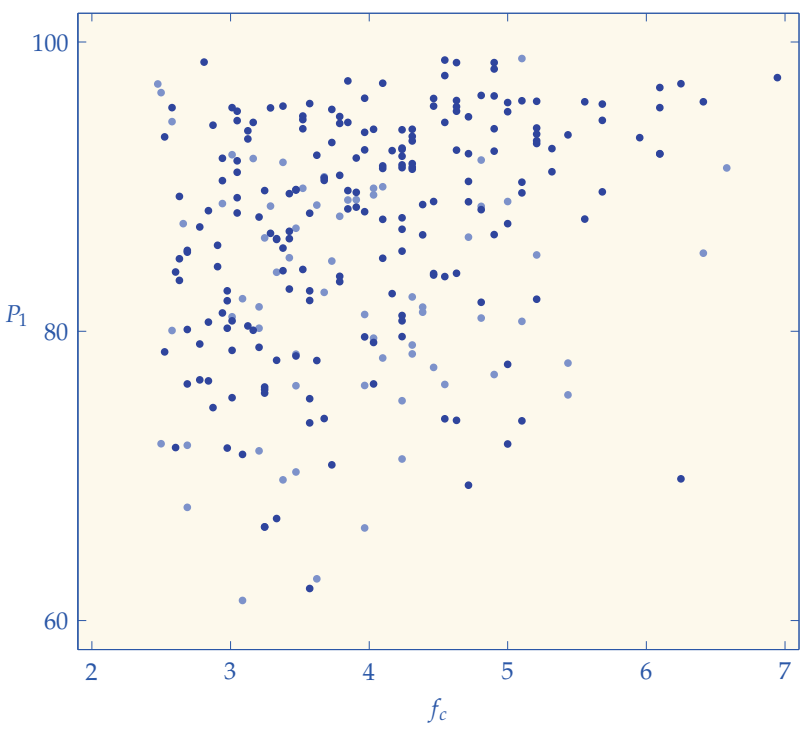

Fig. 7. Relationship between $f_{c}$ and $P_{1}$ for adult VT $(\bullet)$ and paediatric VT $(\bullet)$. There is a weak increase in $P_{1}$ as the heart rate increases. The correlation coefficient is $r=0.28$.

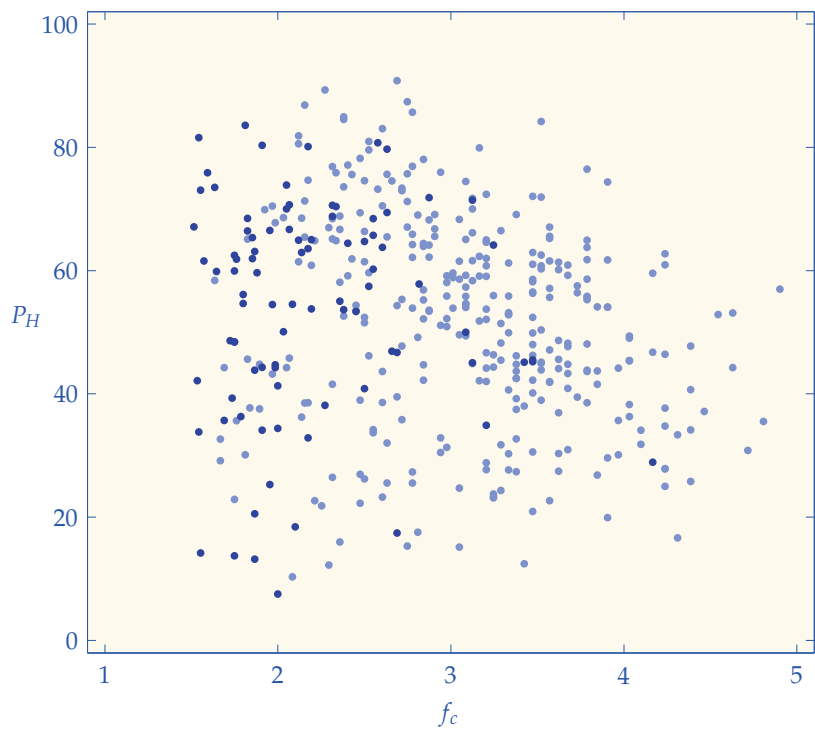

Fig. 8. Relationship between $f_{c}$ and $P_{H}$ for adult SVT $(\bullet)$ and paediatric SVT $(\bullet)$. There is a weak decrease in $P_{H}$ as the heart rate increases. The correlation coefficient is $r=-0.37$. 


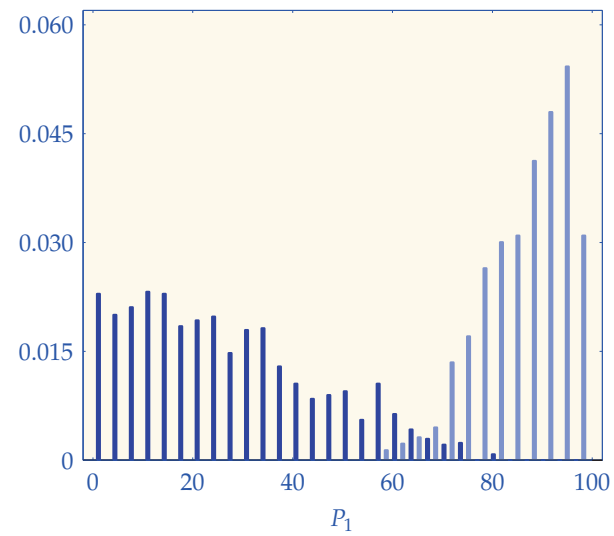

(a) Normalized histogram of the $P_{1}$ parameter.

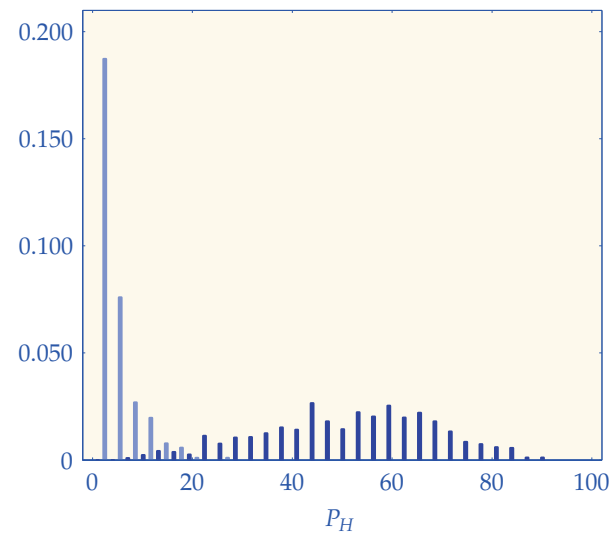

(b) Normalized histogram of the $P_{H}$ parameter.

Fig. 9. Normalized histograms of the discrimination parameters for the SVT records ( $\square$ ) and VT records ( $\square$ ) in the database.

second to test and report the results. All patients within a rhythm type are different in the development database. The test database contains all rhythm repetition from the paediatric database ${ }^{7}$.

The discrimination features were computed for all the $3.2 \mathrm{~s}$ ECG segments of the development database and were linearly combined to fit a logistic regression model. For each segment, we defined the following feature vector:

$$
\boldsymbol{x}=\left\{1, P_{1}, P_{H}\right\} .
$$

Then, the logistic regression model assigned the following probability of being VT to the segment:

$$
\begin{aligned}
& P_{V T}=\frac{1}{1+e^{-Y}}=\frac{1}{1+e^{-\boldsymbol{\beta} \boldsymbol{x}^{T}}}, \\
& Y=\boldsymbol{\beta} \boldsymbol{x}^{T}=\beta_{0}+\beta_{1} P_{1}+\beta_{2} P_{H},
\end{aligned}
$$

where $\beta_{i}$ are the regression coefficients. The decision threshold was set at $P_{V T}=0.5$; i.e., the segment was classified as VT for $P_{V T}>0.5$ or as SVT for $P_{V T} \leq 0.5^{8}$.

Each feature vector was assigned a weight so that all records within a rhythm class contributed equally in the algorithm optimization process, regardless of the number of $3.2 \mathrm{~s}$ segments ${ }^{9}$. The AHA recommendation is more demanding for SVT specificity than for VT sensitivity, so we therefore tripled the total weight of the SVT rhythm category. The weights assigned to a register with $\ell$ segments were:

$$
\omega_{V T, \ell}=\frac{1}{N_{V T} \cdot \ell} \quad \text { and } \quad \omega_{S V T, \ell}=\frac{3}{N_{S V T} \cdot \ell},
$$

\footnotetext{
${ }^{7}$ When a paediatric patient contributed more than one record per rhythm type, the morphologies of the arrhythmias in those records were different; see section 2.2.

8 This is equivalent to VT for $Y>0$ and SVT for $Y \leq 0$.

${ }^{9}$ Records may have one to three segments, depending on their duration.
} 
where $N_{V T}$ and $N_{S V T}$ are the numbers of VT and SVT records in the development database, respectively.

The weighted instances from the development database were used to determine the regression coefficients using Waikato Environment for Knowledge Analysis (WEKA) software(Hall et al., 2009). The regression coefficients, adjusted using 460 SVT segments from 163 records and 303 VT segments from 119 records in the development database, were:

$$
\beta_{o}=-6.000 \quad \beta_{1}=0.145 \quad \beta_{2}=-0.245 \text {. }
$$

Fig. 10 shows the ROC curve of the SVT/VT algorithm for the adult and paediatric records in the test database. The ROC curve depicts the proportion of correctly classified VT segments (sensitivity) against the proportion of wrongly classified SVT segments (1-specificity) as the classification threshold (the value of $Y$ ) varies. The area under the curve (AUC) was 0.999 for the adult database and 0.998 for the paediatric database, which proves the goodness of the classification algorithm for both patient groups.

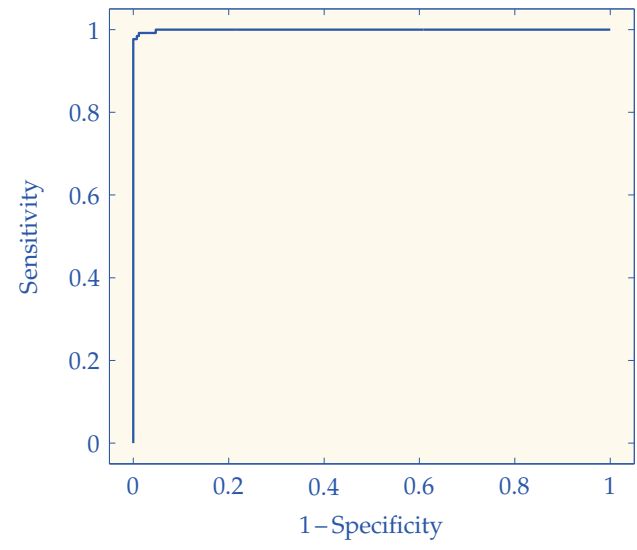

(a) ROC curve for the adult records, AUC $=0.999$.

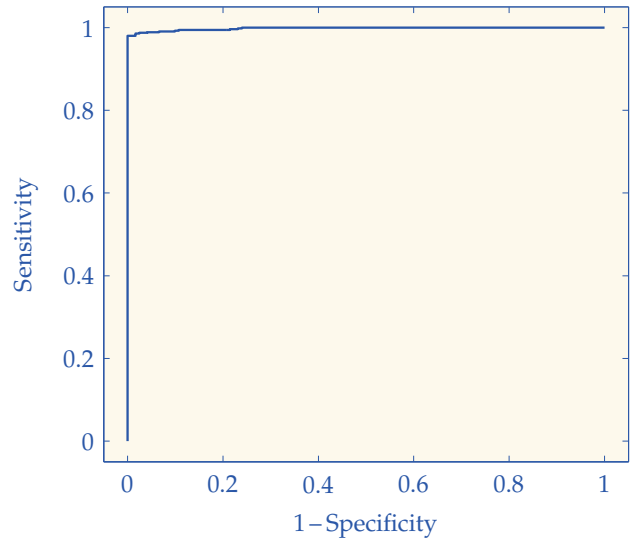

(b) ROC curve for the paediatric records, $\mathrm{AUC}=0.998$.

Fig. 10. Receiver operating charactesistic (ROC) curves for the SVT/VT algorithm for the adult and paediatric records in the test database. AUC=area under the curve.

\subsection{Classification results}

Table 6 shows the classification results for the test database and the corresponding $90 \%$ one-sided CI, estimated using the adjusted Wald interval for binomial proportions. The results for the development database are given per segment and per record. The classification of the record reflects the predominant diagnosis of its segments; when the diagnoses of the segments were balanced, the record was classified as SVT. As shown in table 6, the algorithm meets AHA criteria for SVT and VT classification in both adults and children.

Furthermore, the results are similar when computed per segment and per record. This demonstrates that changes in the dynamics of the arrhythmias in a $9.6 \mathrm{~s}$ analysis interval (record) have very little influence on the spectral features and the classification algorithm. 
SVT and VT can be accurately discriminated using simply a 3.2s ECG segment, i.e. a high-time-resolution algorithm.

\begin{tabular}{|c|c|c|c|c|c|}
\hline \multirow[b]{2}{*}{ Rhythms } & \multicolumn{2}{|c|}{ Segment } & \multicolumn{3}{|c|}{ Record } \\
\hline & number ${ }^{a}$ & Sens/Spec & number ${ }^{a}$ & Sens/Spec ${ }^{b}$ & AHA goal \\
\hline \multicolumn{6}{|l|}{ Adult } \\
\hline SVT & $132(44)$ & 97.0 & $44(44)$ & 97.7 (92.3) & 95 \\
\hline VT & 253 (99) & 100 & 99 (99) & $100(98.0)$ & 75 \\
\hline \multicolumn{6}{|l|}{ Paediatric } \\
\hline SVT & $560(155)$ & 98.8 & $204(155)$ & $98.5(96.9)$ & 95 \\
\hline VT & $121(24)$ & 97.5 & $48(24)$ & $97.9(92.9)$ & 75 \\
\hline \multicolumn{6}{|l|}{ Total } \\
\hline SVT & 692 (199) & 98.4 & 248 (199) & $98.4(97.0)$ & 95 \\
\hline VT & 374 (123) & 99.2 & 147 (123) & $99.3(97.6)$ & 75 \\
\hline
\end{tabular}

Table 6. Classification results per 3.2 s ECG segment and per record for the test database. The SVT/VT algorithm meets American Heart Association (AHA) classification criteria for SVT and VT in both children and adults.

Fig. 11 shows examples of correctly classified high-rate VT and paediatric SVT. SVT with no abnormalities in conduction exhibit narrow QRS complexes, and SVT and VT are accurately discriminated, despite the high rate of the paediatric SVT shown in the examples. When SVT presents abnormalities in conduction leading to wider QRS complexes, or when paediatric VT presents narrow QRS complexes, the discrimination might fail, even for low-rate SVT, as illustrated in the examples shown in Fig. 12.

\section{Discussion and conclusions}

SCD in children is less common than among adults; however, it is less tolerable to families and health care providers. Fatal ventricular arrhythmias have been underappreciated as paediatric problems (Samson et al., 2003). Recent studies show that their incidence varies depending on setting and age (Berg, 2000), and that their presence has been increasingly recognized in both out-of-hospital and in-hospital cardiac arrest (Mogayzel et al., 1995; Nadkarni et al., 2006).

The use of AED in children aged 1-8 years has been recommended since 2003 (Samson et al., 2003), and the European Resuscitation Council Guidelines for Resuscitation integrate the AED in paediatric cardiopulmonary resuscitation (Biarent et al., 2010). The guidelines assume that AED are safe and accurate when used in children older than 1 year and mention that AED are extremely unlikely to advise a shock inappropriately. Nevertheless, the guidelines urge AED purchasers to check that the performance of the AED to be used in children has been tested against paediatric arrhythmias. The 2003 ILCOR recommendation was based on published evidence reporting that AED rhythm analysis algorithms generally satisfied AHA criteria for paediatric rhythms. Two studies (Atkinson et al., 2003; Cecchin et al., 2001) had shown that SAA from adult AED met AHA criteria for VF sensitivity and overall specificity for paediatric rhythms. Their results for paediatric VT were, however, below AHA performance 


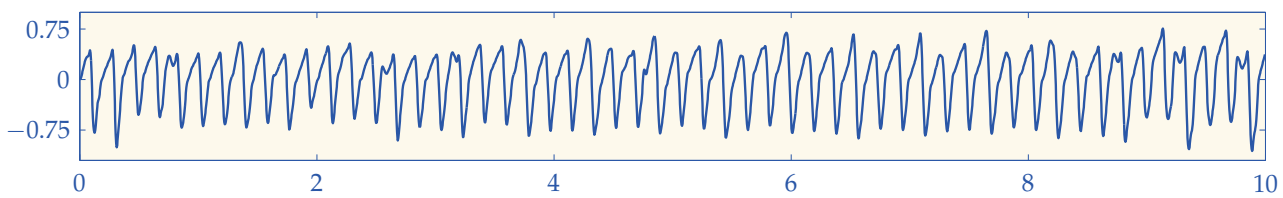

(a) Paediatric VT (heart rate, $326 \mathrm{bpm})$.

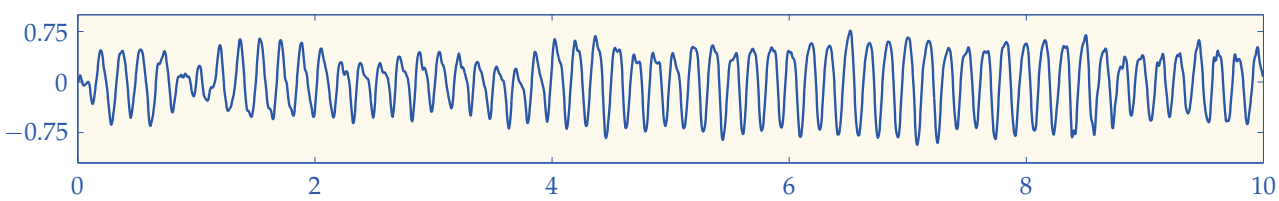

(b) Adult VT (heart rate, $357 \mathrm{bpm}$ ).

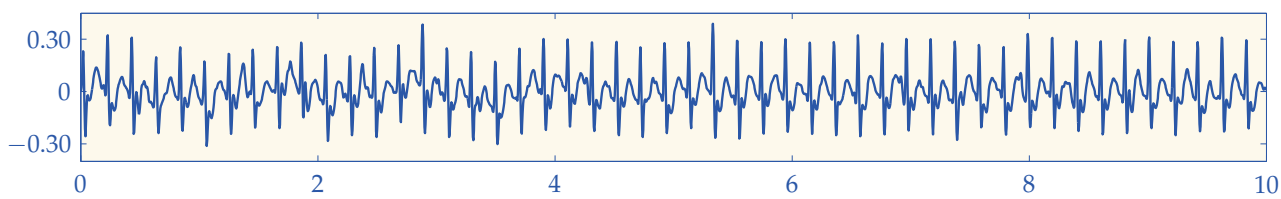

(c) Paediatric SVT (heart rate, $294 \mathrm{bpm}$ ).

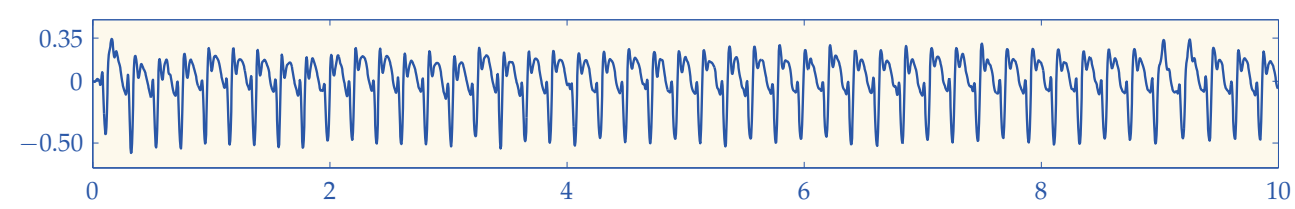

(d) Paediatric SVT (heart rate, $288 \mathrm{bpm}$ ).

Fig. 11. Examples of correctly classified high-rate SVT and VT from the test database. Although rates are high in both cases, VT presents wide QRS complexes

goals in one instance (Cecchin et al., 2001), and tested on only three paediatric VT records in the other instance (Atkinson et al., 2003), so the accurate discrimination of paediatric VT was not addressed. A later study that used a comprehensive database of paediatric SVT and VT demonstrated that an adult SAA failed to identify high-rate paediatric SVT as nonshockable accurately (Atkins et al., 2008). This study proposed the use of specific detection criteria adapted for paediatric use and encouraged adult algorithm verification with paediatric rhythm databases.

Adapting AED SAA to discriminate VF and rapid VT from nonshockable rhythms accurately in children involves two fundamental steps: first, a comprehensive database of paediatric arrhythmias must be compiled to test the algorithm; and second, a reliable SAA for adult and paediatric patients must be developed. Lethal ventricular arrhythmias are rare in children; consequently, the creation of a paediatric database is difficult, particularly for shockable rhythms. In fact, none of the studies in this field has reported an AHA-compliant database for paediatric shockable rhythms (Atkins et al., 2008; Atkinson et al., 2003; Cecchin et al., 2001), and all studies included more than one rhythm per patient and rhythm type. However, adult SAAs accurately detect paediatric VF (Aramendi et al., 2007; Atkins et al., 2008; Atkinson 


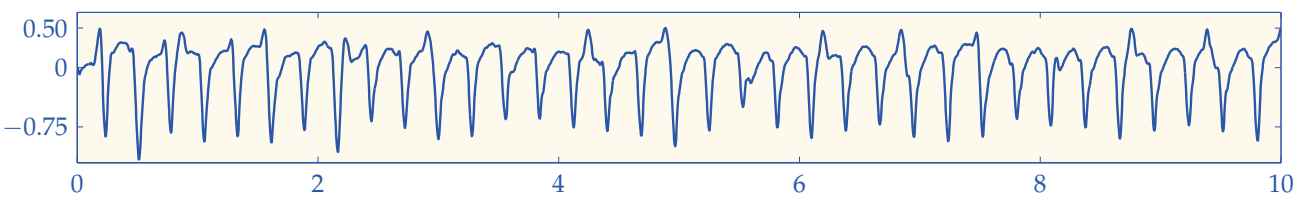

(a) Paediatric VT (heart rate, $217 \mathrm{bpm})$.

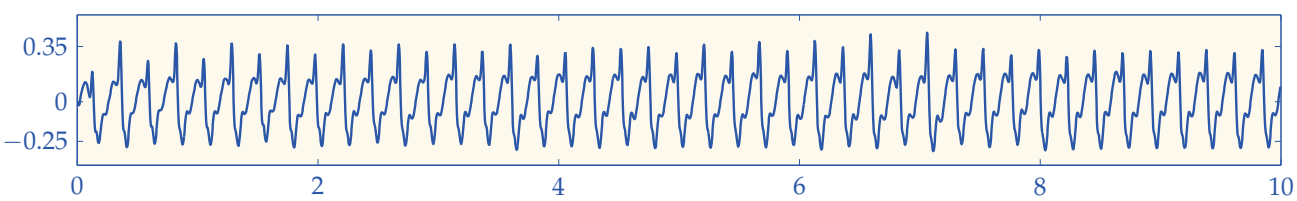

(b) Paediatric SVT (heart rate, $259 \mathrm{bpm}$ ).

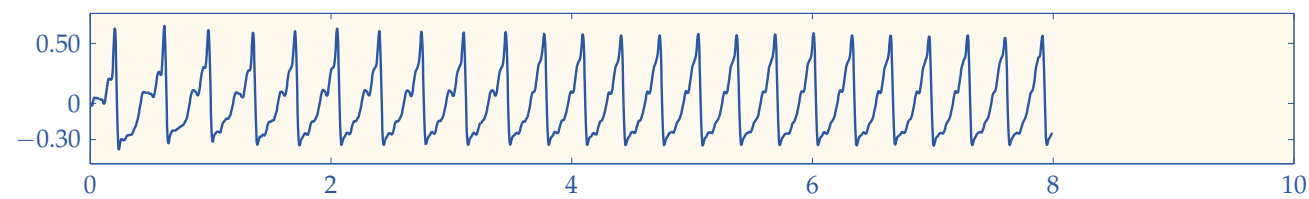

(c) Paediatric SVT (heart rate, $169 \mathrm{bpm}$ ).

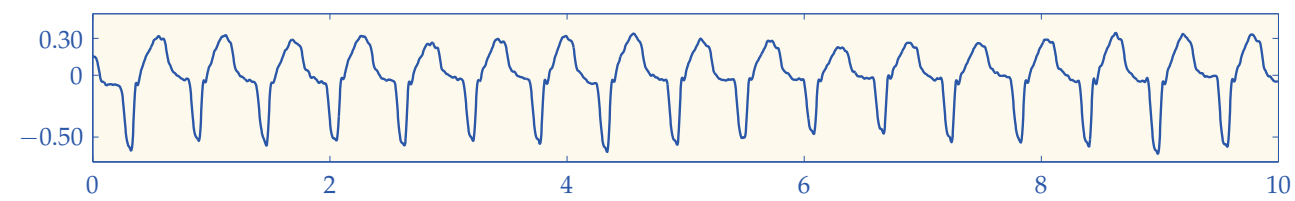

(d) Adult SVT (heart rate, $105 \mathrm{bpm}$ ).

Fig. 12. Examples of misclassified records from the test database.

et al., 2003; Cecchin et al., 2001). The sensitivity for VT and the specificity for SVT of adult SAAs are compromised when high-rate paediatric SVT are included in the databases (Atkins et al., 2008). Consequently, when compiling paediatric rhythms, emphasis should be placed on including a large number of high-rate paediatric SVT and a representative number of paediatric VT. Furthermore, the criteria for the definition of rapid VT depend on the study: $250 \mathrm{bpm}$ in (Cecchin et al., 2001), $200 \mathrm{bpm}$ in (Atkins et al., 2008) and $20 \mathrm{bpm}$ above the age-matched normal rate in (Atkinson et al., 2003). The latter is the most inclusive criterion ${ }^{10}$ and also the most demanding from a SVT/VT discrimination standpoint because lower-rate VTs are included in the databases. The criteria adopted for the definition of rapid VT determine the amount of VT included in the database, and may seriously affect the VT sensitivity and SVT specificity results of the SAA.

SAAs efficiently combine several discrimination parameters obtained from the surface ECG to classify a rhythm as shockable or nonshockable. There exist well-known electrophysiological differences between adult and paediatric rhythms (Chan et al., 2008; Rustwick et al., 2007). Differences between children and adults in both the rate and conduction of VF have been

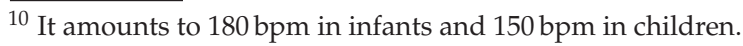


identified, although these differences do not affect VF sensitivity (Cecchin et al., 2001). Heart rates are higher in children than in adults, and SVT occurs more frequently in children whose heart rates often exceed $250 \mathrm{bpm}$ (Atkins et al., 2008). Our data confirm an important overlap in heart rates between paediatric SVT and paediatric and adult VT, which does not occur with adult SVT. Paediatric SVT had significantly higher mean heart rates than did adult SVT (187 bpm vs $131 \mathrm{bpm}, p<0.001$ ). This demonstrates that SAA based on heart rate could either misclassify paediatric SVT or fail to identify paediatric and adult VT accurately, as suggested by Atkins et al. (Atkins et al., 2008). An accurate SVT/VT discrimination must be based on ECG features unaffected by the heart rate. In particular, the distribution of ECG power among the harmonic frequencies is independent of the heart rate, as shown in section 3 . We have designed a robust SVT/VT algorithm based on these features that accurately discriminates between SVT and VT in paediatric and adult patients. Furthermore, the algorithm needs only $3.2 \mathrm{~s}$ of the ECG to discriminate between the arrhythmias.

An accurate SVT/VT discrimination algorithm can be integrated in current adult SAA to address the classification problems posed by high-rate paediatric SVT. Other strategies, ranging from the use of unmodified adult SAA (Atkinson et al., 2003; Cecchin et al., 2001) to the definition of paediatric-specific thresholds Atkins et al. (2008), have also been proposed ${ }^{11}$. Using the strategy proposed in this study, the same AED algorithm can be applied to paediatric and adult patients. The SVT/VT algorithm would be integrated in the SAA as indicated in Fig. 13, which shows the block diagram for a general SAA design. Rhythm identification can be structured in three stages. First, asystole is detected based on the amplitude or energy of the ECG. Then, nonshockable and shockable rhythms are discriminated. Finally, a VF/VT discrimination algorithm is needed, for two reasons: VT can be better treated using cardioversion therapy rather than a defibrillation shock, and VT is shocked based on heart rate. It is precisely at this stage that the SVT/VT discrimination algorithm described in section 4 should be incorporated.

To conclude, we have shown how a SVT/VT discrimination algorithm based on spectral features can be designed and incorporated into an adult SAA to make its use safe for children.

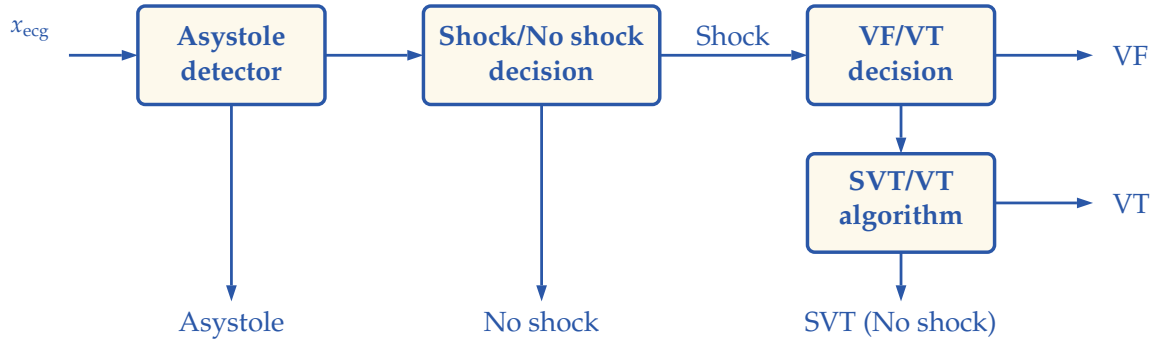

Fig. 13. General block diagram of a shock advice algorithm (SAA). The addition of the SVT/VT algorithm addresses the problem of the accurate detection of high-rate paediatric SVT, and the SAA can therefore be safely used both in adults and children.

${ }^{11}$ Paediatric and adult thresholds can be independently set in an AED because the AED must be aware of the type of patient in order to adjust the defibrillation energy dose. 


\section{References}

Appleton, G. O., Cummins, R. O., Larson, M. P. \& Graves, J. R. (1995). CPR and the single rescuer: at what age should you "call first" rather than "call fast"?, Annals of Emergency Medicine 25(4): 492-494.

Aramendi, E., Irusta, U., de Gauna, S. et al. (2006). Comparative analysis of the parameters affecting aed specificity: Pediatric vs. adult patients, Computers in Cardiology, 2006, pp. $445-448$.

Aramendi, E., Irusta, U., Pastor, E. et al. (2010). ECG spectral and morphological parameters reviewed and updated to detect adult and paediatric life-threatening arrhythmia., Physiological Measurement 31(6): 749-761.

Aramendi, E., Irusta, U., Ruiz de Gauna, S. \& Ruiz, J. (2007). Comparative analysis of the parameters affecting AED rhythm analysis algorithm applied to pediatric and adult ventricular tachycardia, Computers in Cardiology, 2007, pp. 419-422.

Atkins, D. L., Hartley, L. L. \& York, D. K. (1998). Accurate recognition and effective treatment of ventricular fibrillation by automated external defibrillators in adolescents, Pediatrics 101(3 Pt 1): 393-397.

Atkins, D. L., Scott, W. A., Blaufox, A. D. et al. (2008). Sensitivity and specificity of an automated external defibrillator algorithm designed for pediatric patients., Resuscitation 76(2): 168-174.

Atkinson, E., Mikysa, B., Conway, J. A. et al. (2003). Specificity and sensitivity of automated external defibrillator rhythm analysis in infants and children., Ann Emerg Med 42(2): 185-196.

Barro, S., Ruiz, R., Cabello, D. \& Mira, J. (1989). Algorithmic sequential decision-making in the frequency domain for life threatening ventricular arrhythmias and imitative artefacts: a diagnostic system., J Biomed Eng 11(4): 320-328.

Berg, R. A. (2000). Paediatric sudden death, Best Practice \& Research Clinical Anaesthesiology 14(3): 611-624.

Biarent, D., Bingham, R., Eich, C. et al. (2010). European Resuscitation Council Guidelines for Resuscitation 2010 Section 6. Paediatric life support., Resuscitation 81(10): 1364-1388.

Cecchin, F., Jorgenson, D. B., Berul, C. I. et al. (2001). Is arrhythmia detection by automatic external defibrillator accurate for children?: Sensitivity and specificity of an automatic external defibrillator algorithm in 696 pediatric arrhythmias., Circulation 103(20): 2483-2488.

Chan, T. C., Sharieff, G. Q. \& Brady, W. J. (2008). Electrocardiographic manifestations: pediatric ECG., J Emerg Med 35(4): 421-430.

de Luna, A. B., Coumel, P. \& Leclercq, J. F. (1989). Ambulatory sudden cardiac death: Mechanisms of production of fatal arrhythmia on the basis of data from 157 cases, American Heart Journal 117(1): 151-159.

Hall, M., Frank, E., Holmes, G. et al. (2009). The weka data mining software: An update, ACM SIGKDD Explorations Newsletter 11(1): 10-18.

Irusta, U. \& Ruiz, J. (2009). An algorithm to discriminate supraventricular from ventricular tachycardia in automated external defibrillators valid for adult and paediatric patients., Resuscitation 80(11): 1229-1233.

Irusta, U., Ruiz, J., Aramendi, E. \& de Gauna, S. R. (2008). Amplitude, frequency and complexity features in paediatric and adult ventricular fibrillation., Resuscitation 77: S53-S53. 
Jacobs, I., Nadkarni, V., Bahr, J. et al. (2004). Cardiac arrest and cardiopulmonary resuscitation outcome reports: update and simplification of the Utstein templates for resuscitation registries., Circulation 110(21): 3385-3397.

Kerber, R. E., Becker, L. B., Bourland, J. D. et al. (1997). Automatic external defibrillators for public access defibrillation: recommendations for specifying and reporting arrhythmia analysis algorithm performance, incorporating new waveforms, and enhancing safety. A statement for health professionals from the American Heart Association Task Force on Automatic External Defibrillation, Subcommittee on AED Safety and Efficacy., Circulation 95(6): 1677-1682.

Larsen, M. P., Eisenberg, M. S., Cummins, R. O. \& Hallstrom, A. P. (1993). Predicting survival from out-of-hospital cardiac arrest: a graphic model., Ann Emerg Med 22(11): 1652-1658.

Luu, M., Stevenson, W., Stevenson, L., Baron, K. \& Walden, J. (1989). Diverse mechanisms of unexpected cardiac arrest in advanced heart failure, Circulation 80(6): 1675-1680.

Manole, M. D. \& Saladino, R. A. (2007). Emergency department management of the pediatric patient with supraventricular tachycardia, Pediatric Emergency Care 23(3): 176-185.

Mogayzel, C., Quan, L., Graves, J. R. et al. (1995). Out-of-hospital ventricular fibrillation in children and adolescents: causes and outcomes, Annals of Emergency Medicine 25(4): 484-491. PMID: 7710153.

Myerburg, R. J. (2001). Sudden cardiac death: exploring the limits of our knowledge., J Cardiovasc Electrophysiol 12(3): 369-381.

Nadkarni, V. M., Larkin, G. L., Peberdy, M. A. et al. (2006). First documented rhythm and clinical outcome from in-hospital cardiac arrest among children and adults, JAMA: The Journal of the American Medical Association 295(1): 50-57.

Ruiz de Gauna, S., Ruiz, J., Irusta, U. \& Aramendi, E. (2008). Parameters affecting shock decision in pediatric automated defibrillation., Proc. Computers in Cardiology, pp. 929-932.

Rustwick, B., Geheb, F., Brewer, J. \& Atkins, D. (2007). Electrocardiographic characteristics for automated external defibrillator algorithms are different between children and adults., Circulation 116.

Samson, R. A., Berg, R. A., Bingham, R. et al. (2003). Use of automated external defibrillators for children: an update: an advisory statement from the pediatric advanced life support task force, International Liaison Committee on Resuscitation., Circulation 107(25): 3250-3255.

Schwartz, P. J., Garson, A., Paul, T. et al. (2002). Guidelines for the interpretation of the neonatal electrocardiogram. A task force of the European Society of Cardiology., Eur Heart J 23(17): 1329-1344.

Sirbaugh, P. E., Pepe, P. E., Shook, J. E. et al. (1999). A prospective, population-based study of the demographics, epidemiology, management, and outcome of out-of-hospital pediatric cardiopulmonary arrest., Ann Emerg Med 33(2): 174-184.

Strohmenger, H. U., Lindner, K. H., Keller, A. et al. (1996). Spectral analysis of ventricular fibrillation and closed-chest cardiopulmonary resuscitation., Resuscitation 33(2): 155-161.

Valenzuela, T. D., Roe, D. J., Cretin, S. et al. (1997). Estimating effectiveness of cardiac arrest interventions: a logistic regression survival model., Circulation 96(10): 3308-3313. 


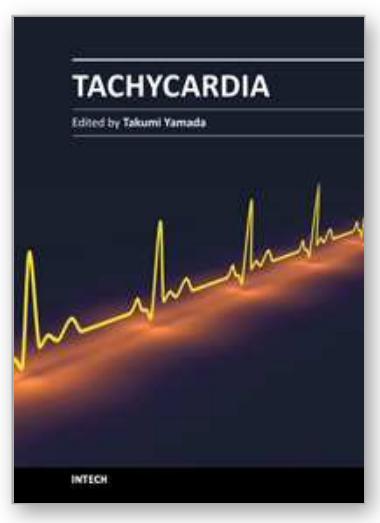

\author{
Tachycardia \\ Edited by Prof. Takumi Yamada
}

ISBN 978-953-51-0413-1

Hard cover, 202 pages

Publisher InTech

Published online 30, March, 2012

Published in print edition March, 2012

Heart rates are normally controlled by a natural pacemaker, the sinus node, and normal heart rhythm is called sinus rhythm. Tachycardia is defined as a faster heart rhythm than normal sinus rhythm. Tachycardias can cause symptoms such as palpitations, chest pain, shortness of breath and fatigue, which reduce the quality of life. Fast tachycardias can cause hemodynamic collapse and sudden cardiac death. The causes, mechanisms, and origins of tachycardias are various. The diagnosis of tachycardias is made by electrocardiograms and electrophysiological testing. Tachycardias can be managed and treated by pharmacological and nonpharmacological approaches. This book covers these concerns from basic and clinical points of view and will lead to a further understanding and improvement in the clinical outcomes of tachycardias.

\title{
How to reference
}

In order to correctly reference this scholarly work, feel free to copy and paste the following:

U. Irusta, E. Aramendi, J. Ruiz and S. Ruiz de Gauna (2012). Accurate Detection of Paediatric Ventricular Tachycardia in AED, Tachycardia, Prof. Takumi Yamada (Ed.), ISBN: 978-953-51-0413-1, InTech, Available from: http://www.intechopen.com/books/tachycardia/accurate-detection-of-pediatric-ventricular-tachycardia-inaed

\section{INTECH}

open science | open minds

\section{InTech Europe}

University Campus STeP Ri Slavka Krautzeka 83/A 51000 Rijeka, Croatia Phone: +385 (51) 770447 Fax: +385 (51) 686166 www.intechopen.com

\section{InTech China}

Unit 405, Office Block, Hotel Equatorial Shanghai No.65, Yan An Road (West), Shanghai, 200040, China 中国上海市延安西路65号上海国际贵都大饭店办公楼 405 单元 Phone: +86-21-62489820

Fax: $+86-21-62489821$ 
(C) 2012 The Author(s). Licensee IntechOpen. This is an open access article distributed under the terms of the Creative Commons Attribution 3.0 License, which permits unrestricted use, distribution, and reproduction in any medium, provided the original work is properly cited. 